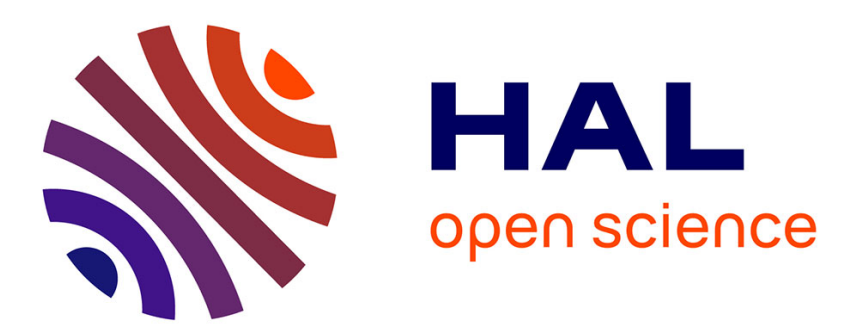

\title{
Light and electric field induced unusual large-scale charge separation in hybrid semiconductor objects
}

\author{
Ambrose Melvin, Eric Lebraud, Patrick Garrigue, Alexander Kuhn
}

\section{To cite this version:}

Ambrose Melvin, Eric Lebraud, Patrick Garrigue, Alexander Kuhn. Light and electric field induced unusual large-scale charge separation in hybrid semiconductor objects. Physical Chemistry Chemical Physics, 2020, 10.1039/D0CP03262J . hal-02938181

\section{HAL Id: hal-02938181 \\ https://hal.science/hal-02938181}

Submitted on 22 Sep 2020

HAL is a multi-disciplinary open access archive for the deposit and dissemination of scientific research documents, whether they are published or not. The documents may come from teaching and research institutions in France or abroad, or from public or private research centers.
L'archive ouverte pluridisciplinaire HAL, est destinée au dépôt et à la diffusion de documents scientifiques de niveau recherche, publiés ou non, émanant des établissements d'enseignement et de recherche français ou étrangers, des laboratoires publics ou privés. 


\title{
Light and electric field induced unusual large-scale charge separation in hybrid semiconductor objects
}

\author{
Ambrose A. Melvin1, Eric Lebraud2,3, Patrick Garrigue1, Alexander Kuhn1*
}

Separation of electric charges is the most crucial phenomenon in natural photosynthesis, and also extremely important for many artificial energy conversion systems based on semiconductors. The usual roadblock in this context is the fast recombination of electrons and holes. Here we demonstrate that the synergy of light and electric fields allows separating very efficiently electric charges over an unusually large distance in TiO2. The generated internal electric field can also be used to shuttle electrons simultaneously to the two opposite sides of a hybrid TiO2-polyaniline object. This counterintuitive behavior is based on the combination of the principles of bipolar electrochemistry and semi-conductor physics.

Light-induced charge separation has received considerable attention due to its tremendous importance not only in natural systems such as photosynthesis, but especially also for developing new approaches related to renewable energy conversion and storage systems.1-5 Charge carrier generation and separation has been studied and optimized in the context of various applications ranging from photocatalysis1, 6, 7 and photoelectrocatalysis6, 8 to photovoltaics. 9 In photocatalysis, charge generation is induced by the absorption of light with an appropriate wavelength by semiconducting materials such as $\mathrm{TiO} 2, \mathrm{ZnO}, \mathrm{MnO} 2, \mathrm{Fe} 2 \mathrm{O} 3$ and $\mathrm{CdS} 10-12$ The electrons and holes are then separated and tend to move towards the surface in order to perform catalytic reactions. However, one of the biggest problems in such systems is the high probability of charge recombination. Charge separation under the influence of light, electric or magnetic fields, and combinations thereof has been studied, both from an experimental and theoretical point of view.1317 Electric field induced charge separation is well understood in the case of bipolar electrochemistry and extensively used e.g. for the purpose of functional material synthesis and related topics.18-21 In this case, a conducting substrate is placed in a cell filled with electrolyte together with two feeder electrodes, allowing to apply a potential difference. The presence of a potential gradient in the electrolyte will polarize the substrate with respect to the surrounding solution and if this polarization is big enough it will act as a bipolar electrode with two opposite redox reactions (oxidation and reduction) occurring at the polarized extremities.18, 20, 22-24 This break of symmetry results in the generation of anisotropic Janus objects by a site selective electrodeposition process. However, this concept works best for good conductors, having a lower resistance than the solution, and cannot be applied directly to semiconductors, especially metal oxides, due to their low conductivity. Therefore, light is required in addition to generate a sufficient amount of charge carriers, enhancing the object's conductivity. The simultaneous presence of an electric field in the solution helps to separate the generated charges and allows performing bipolar electrochemistry with the polarized object. This approach was first reported by Ongaro et al. for the site selective deposition of Au on $\mathrm{TiO}_{2}$ nanofibres. 25 Here the electric field helps the charges to move to the extremities, resulting in electric field assisted photochemistry. Such Janus-type particles, combining metal oxides and metals, have gained considerable interest in recent years due to their multifunctional features, especially with respect to catalysis.26-28 An analogue example of TiO2-metal Janus particles has been reported by Tiewcharoen et al. where TiO2 nano- and microparticles were placed in an electric field in the presence of light for anisotropic electrodeposition.29 Interestingly, the direction of polarization was reported to be the opposite of what is observed when performing bipolar electrochemistry with a normal conductor. However, the origin of this reverse polarization is so far not well understood. 
Here we study in detail the underlying mechanism of such a reverse polarization of $\mathrm{TiO} 2$ and demonstrate that it can occur at unusually large scales, up to the range of centimetres. Furthermore, the effect is exploited for directing electrons simultaneously to both extremities of a hybrid bipolar object, composed of the semiconductor and a polyaniline composite. This counterintuitive behaviour results from the synergy between the principles of bipolar electrochemistry and semiconductor physics. It allows fine-tuning the pathway of the light-generated charge carriers in order to obtain either uni- or bi-directional electron transfer. The charge transfer is revealed by the reductive deposition of gold nanoparticles, but might be generalized to any other type of redox reaction which fits to the band gap energy of the semiconductor. This opens various applications, from photovoltaics to water splitting and the synthesis of multifunctional particles and objects.

An open bipolar electrochemical set-up was used for the wireless electrodeposition of gold on the surface of TiO2 pellets as illustrated in Scheme 1a. The TiO2 pellet was placed in the centre of the cell and acted as a bipolar electrode. Two feeder electrodes were used to apply the global electric field. The UV lamp was placed above the centre compartment of the bipolar cell for the purpose of photoirradiation, as illustrated in Figure S1. When applying simultaneously both, the electric field and light, the $\mathrm{TiO} 2$ pellet gets polarized at its extremities ( $\delta+$ and $\delta$-) with respect to the surrounding solution. Usually, in the case of bipolar electrochemistry involving a classic conductor such as metal or carbon, the bipolar object itself is equipotential, and therefore the reduction process, e.g. of metal ions, occurs at the $\delta$ - extremity.18, 24, 30, 31 Interestingly, in the present case gold deposition is observed at the $\delta+$ site (Figure $1 \mathrm{~b}$ ). The amount of deposited gold is much higher at the $\delta+$ site as revealed by SEM images (Figure $1 \mathrm{f}$ and Figure $\mathrm{S} 2 \mathrm{~b}$ ). This is absolutely counterintuitive with respect to normal bipolar electrochemistry. The origin of this unusual phenomenon can be explained by the generation of an internal electric field inside the semiconductor, which, in contrast to a conductor, is not an equipotential object. The internal field drives electrons from the $\delta$ - to the $\delta+$ side (arrow in Scheme $1 \mathrm{a})$, where they become available for metal ion reduction. This process is only possible in the presence of light and is more pronounced in the case of photosensitive semiconductors, especially for the purpose of efficient photocatalytic water splitting. 32 The generation of such a built-in electric field is usually conditioned by the presence of a heterojunction between two different Fermi levels, as shown in Scheme $1 \mathrm{~b}$. When applying light and an electric field, band bending occurs, inducing a separation of the photogenerated charge carriers (electrons and holes).The electron deficient part of TiO2 (blue line in Scheme 1b) pulls the electrons away from the electron rich side of TiO2 (red lineScheme $1 b$ ). This separation of charge carriers is sufficient enough for Au deposition to occur at the $\delta+$ side. In the case of materials without heterojunction, charge flow occurs without band bending. Hence, $\delta$ - remains the site for metal ion reduction (Figure 1c). A series of experiments was performed to understand and validate the existence of an internal electric field, revealed by the unusual location of Au electrodeposition. Bipolar electrodeposition was carried out by varying the electric field and light intensity in order to study their respective impact on the efficiency of charge generation and charge separation. First, the relative light intensity was increased from 0 to 1 percent in the presence of an electric field of $90 \mathrm{~V} / \mathrm{cm}$. In the absence of light, absolutely no electrodeposition is visible on the TiO2 pellet (Figure 1a). When increasing the light intensity to $1 \%$, perfectly asymmetric Au deposition occurs, indicating an efficient charge separation at the centimeter scale (Figure 1b). The reduction of the gold ions is most likely compensated by the thermodynamically favorable oxidation of water at the opposite extremity. Preventing charge carrier recombination at such a large scale is rather unusual, as charge separation and recombination is 
typically occurring at the nanometer scale (1-100 nm).33, 34 Maintaining a light intensity of $1 \%$, but without applying an electric field, results in a fully homogenous coverage of the TiO2 surface with gold nanoparticles as the energy level of $\mathrm{Au3}+$ reduction falls between the valence and conduction band of $\mathrm{TiO}_{2}$ (Figure S3). In this case, the light induced charge carriers are immediately reacting at the location of their production, indicating that the absence of electric field doesn't allow a large scale separation of electrons and holes. In order to further enhance the strength of polarization and the amount of free charge carriers, the intensity of light and of the electric field has been increased. With an electric field of $120 \mathrm{~V} / \mathrm{cm}$ and $5 \%$ light intensity, the area covered with gold is almost double (Figure 1d) compared to the previous conditions (Figure 1b). However, increasing only the electric field, but keeping $1 \%$ light intensity does not change the extension and efficiency of $\mathrm{Au}$ electrodeposition (results not shown). This infers that even at a lower electric field, charge separation is efficient enough to generate a large-scale asymmetry and is not the limiting parameter for the observed effect. Microscope images of the two opposite extremities of the TiO2 pellet surface, i.e. $\delta+$ and $\delta$ - side, clearly reveal a very dense population of bright Au particles on the $\delta+$ part, whereas the $\delta$ - part shows no significant metal deposition (Figure $1 \mathrm{f}$ and 1 e respectively, as well as Figure S2). This illustrates the reverse polarization of the $\mathrm{TiO} 2$ pellet by the simultaneous presence of light and an electric field.

The main physical factors that can influence the charge separation are electric, optical and magnetic fields. However, an important additional parameter is also the chemical nature and structure of the semiconductor. In order to facilitate light induced charge separation, three types of structural features have been identified as being important: $p$-n junctions, polar surface terminations and polymorph junctions.32 TiO2 is an n-type semiconductor and free of any functionalization, doping and mixing with other metal oxide. Therefore, only polymorph junctions, due to the presence of different crystal phases of $\mathrm{TiO}_{2}$, can have an impact on the charge separation efficiency. In order to better understand this aspect, we studied Au electrodeposition on pure phases of TiO2 i.e. anatase$\mathrm{TiO} 2$ (A-TiO2) and rutile-TiO2 ( $\mathrm{R}-\mathrm{TiO} 2$ ), as well as a mixed phase $\mathrm{TiO} 2$ i.e. anatase+rutile $\mathrm{TiO} 2$ (ARTiO2) with a 50:50\% content of both phases. The crystallographic properties of the individual TiO2 pellets have been characterized by XRD measurements (supporting information S4). The results confirm that pressing the initial pure powders into pellets does not alter their crystal structure, despite the high applied pressure.

Electrodeposition of Au was observed on the $\delta$ - side in the case of the pure phases (Figures 2a and $2 \mathrm{c}$ ) and on the $\delta+$ extremity for the mixed phase (Figure $2 \mathrm{~b}$ ). In the case of the pure phase materials, charge separation is analogue to what is known for conventional bipolar electrochemistry. In contrast, for the mixed phase it is reasonable to assume that charge separation is facilitated by the heterojunctions between the different phases in the presence of the electric field. This is further confirmed by carrying out the same type of experiment with other pure phase semiconductors. Metal oxides and semiconductors with metallic properties i.e. Ta2O5 and doped silicon were used for this purpose. In both cases, no heterojunctions are present and consequently the electrodeposition occurs at the usual $\delta$ - extremity.

Based on these results, we were interested in checking whether a hybrid system, composed of a conducting material and a semiconductor, might allow moving electrons simultaneously in both directions, visualized by the reduction of metal ions at the two extremities. Two materials were selected as constituents of such a hybrid object, i.e. the mixed phase of TiO2 (AR-TiO2) and a blend of $70 \mathrm{wt} \%$ polyaniline (PANi) with $30 \mathrm{wt} \%$ polyvinyl chloride-carboxylated (PVC-carboxy). The blending helped increasing the mechanical strength of the final PANi film.35 
While casting the polymer film, the AR-TiO2 pellet is embedded into the blend and left to dry. The band structure of both materials is shown in Figure 3d. Both, PANi and AR-TiO2 are semiconductors, however the doped form of PANi has a very high intrinsic conductivity, close to metallic conductors, whereas AR-TiO2 behaves as a photoconductor with a rather low conductivity. Since PANi does not have heterojunctions, its polarization in the electric field is analogue to a normal conductor. Consequently, and in agreement with the principles of classic bipolar electrochemistry, metal ion reduction occurs at the $\delta$ - extremity of the polymer (Figure 3a). However, the AR-TiO2 pellet, embedded in the polymeric matrix, shows the opposite behavior, as described above. Therefore, metal deposition is observed at the $\delta+$ extremity of the AR-TiO2 (Figure 3a). Interestingly, at the latter extremity, electrons can even spill over from AR-TiO2 to PANi, due to the favorable band energies (Figure $3 \mathrm{~d}$ ). The conduction band of PANi lies below the one of AR-TiO2,36 allowing an electron transfer from AR-TiO2 to PANi, resulting in metal deposition also at the $\delta+$ extremity of the polymer (Figure $3 \mathrm{~b}$ ). This is the direct evidence that in the presence of the electric field and light, electrons are shuttled inside the hybrid conductor simultaneously to both extremities. Another evidence of charge transfer at the junction between $\mathrm{TiO} 2$ and polymer is provided by I-V curve recordings (Figure S5). When contacting both extremities (TiO2 on one side and the polymer on the other side) a typical diode type behavior can be observed. Additionally, the presence of UV light causes a significant increase in current compared to the measurement without light. The completely counterintuitive observation that electrons can trigger metal ion reduction in parallel at both opposite ends of the hybrid structure is unique and results from the synergy of the principles of bipolar electrochemistry and semiconductor physics. Electron microscopy images clearly indicate the presence of bright Au particles, not only at the $\delta$ - extremity, as expected in a normal bipolar experiment, but as well on the $\delta+$ side. There, gold is visible on both, the AR-TiO2 and the PANi/PVCcarboxy surface, which is the consequence of a spillover of electrons from AR-TiO2 to the conducting polymer. An important question concerns also the location of the counter reaction, that means the oxidation of water. The possible sites for $\mathrm{O} 2$ evolution are indicated in Figure $\mathrm{S6}$. On the left side of the pellet, oxygen is generated via a photoelectrochemical process in order to provide the adequate amount of electrons necessary for the gold salt reduction occurring in the right side of the semiconductor pellet. The second site for oxygen formation is on the right side of the conducting polymer were the bipolar object has a positive polarization. Therefore, oxygen is formed simultaneously at both locations because different mechanisms are involved (photoelectrochemical and bipolar electrochemical pathways). This unusual behavior is the witness of electron transfer occurring along both directions of the imposed electric field.

We have successfully demonstrated that the simultaneous presence of light and an electric field can trigger an efficient charge carrier separation in semiconductors at an exceptionally large scale. In the special case of mixed phase $\mathrm{TiO} 2$, the presence of different crystal phases induces heterojunctions in the semiconducting material matrix, allowing an inversion of the global direction of electron transfer. This can be revealed by the reduction of gold ions, occurring at the $\delta+$ extremity of the polarized object, instead of the usual $\delta$ - side. Other, less noble metals might be also formed by this mechanism, however eventually in competition with oxygen reduction. ORR occurs at a more negative potential than Au3+ reduction from a thermodynamic point of view. Thus, at first sight, the present concept might only work for metals with rather positive formal potentials. However, like in classic electrodeposition, even metals with lower reduction potentials like $\mathrm{Zn}, \mathrm{Ni}$ and $\mathrm{Cu}$ can still be reduced in parallel to oxygen (and for $\mathrm{Zn}$ even in parallel to the thermodynamically much more favourable $\mathrm{H}+$ reduction), due to kinetic aspects.

The presented hybrid objects composed of a semiconductor, having mixed crystallographic phases and low conductivity, and a metallic semiconductor, show an even more unusual behaviour. In this 
case, electrons can be transferred to the metal precursor in a symmetric way, which means at both ends of the object. Such a bidirectional transport of charge carriers is reported for the first time and is the result of the interplay between the principles of bipolar electrochemistry and semiconductor physics. We anticipate that this exotic behaviour might find interesting applications in various fields ranging from photovoltaics and photoelectrocatalysis to the synthesis of multifunctional materials.

\section{Conflicts of interest}

There are no conflicts to declare.

Acknowledgments

The work has been funded by the European Research Council (ERC) under the European Union's Horizon 2020 research and innovation program (grant agreement $n^{\circ} 741251$, ERC Advanced grant ELECTRA). The work is also supported by funding from Campus France under the PRESTIGE postdoctoral fellowship program. The authors acknowledge technical support from Philippe Vinatier for preparing the semiconductor pellets.

\section{References}

1. Y. Bai, Y. Zhou, J. Zhang, X. Chen, Y. Zhang, J. Liu, J. Wang, F. Wang, C. Chen, C. Li, R. Li and C. Li, ACS Catal., 2019, 9, 3242-3252.

2. G. J. Hedley, A. Ruseckas and I. D. W. Samuel, Chem. Rev., 2017, 117, 796-837.

3. S. Karlsson, J. Boixel, Y. Pellegrin, E. Blart, H.-C. Becker, F. Odobel and L. Hammarström, J. Am. Chem. Soc., 2010, 132, 17977-17979.

4. T. Tatsuma, H. Nishi and T. Ishida, Chem. Sci., 2017, 8, 3325-3337.

5. M. Frank, J. Ahrens, I. Bejenke, M. Krick, D. Schwarzer and G. H. Clever, J. Am. Chem. Soc., 2016, 138, 8279-8287.

6. Q. Wang and K. Domen, Chem. Rev., 2020, 120, 919-985.

7. T. Takata, J. Jiang, Y. Sakata, M. Nakabayashi, N. Shibata, V. Nandal, K. Seki, T. Hisatomi and K. Domen, Nature, 2020, 581, 411-414.

7. J. Schneider, M. Matsuoka, M. Takeuchi, J. Zhang, Y. Horiuchi, M. Anpo and D. W. Bahnemann, Chem. Rev., 2014, 114, 9919-9986.

8. K. Nakano, Y. Chen, B. Xiao, W. Han, J. Huang, H. Yoshida, E. Zhou and K. Tajima, Nat. Commun., 2019, 10, 2520.

9. S. Wang, G. Liu and L. Wang, Chem. Rev., 2019, 119, 5192-5247.

10. K. Maeda and K. Domen, J. Phys. Chem. Lett., 2010, 1, 2655-2661.

11. A. A. Melvin, K. Illath, T. Das, T. Raja, S. Bhattacharyya and C. S. Gopinath, Nanoscale, 2015, 7, 13477-13488.

12. X. Wang and C. Li, J. Phys. Chem. C, 2018, 122, 21083-21096.

13. J.-L. Wang, H.-J. Jiang, Z. He, J.-W. Liu, R. Wang, W.-R. Huang, L.-T. Feng, X.-F. Ren, Z.-H. Hou and S.-H. Yu, Nano Lett., 2020, 20, 2763-2769. 
14. F. Aslan, G. Adam, P. Stadler, A. Goktas, I. H. Mutlu and N. S. Sariciftci, Sol. Energy, 2014, 108, 230-237.

15. K. Nakata and A. Fujishima, J. Photoch. Photobio C:, 2012, 13, 169-189.

16. A. Goktas, A. Tumbul, Z. Aba and M. Durgun, Thin Solid Films, 2019, 680, 20-30.

17. S. E. Fosdick, K. N. Knust, K. Scida and R. M. Crooks, Angew. Chem. Int. Ed., 2013, 52, 1043810456.

18. G. Loget, D. Zigah, L. Bouffier, N. Sojic and A. Kuhn, Acc. Chem. Res., 2013, 46, 2513-2523.

19. N. Shida, Y. Zhou and S. Inagi, Acc. Chem. Res., 2019, 52, 2598-2608.

20. P. Arenas-Guerrero, Á. V. Delgado, A. Ramos and M. L. Jiménez, Langmuir, 2019, 35, 687-694.

21. L. Koefoed, S. U. Pedersen and K. Daasbjerg, Curr. Opin. Electrochem., 2017, 2, 13-17.

22. I. Malytska, T. Doneux, M. Bougouma, A. Kuhn and L. Bouffier, J. Phys. Chem. C, 2018. 123, 5647-5652.

23. Z. A. Fattah, L. Bouffier and A. Kuhn, Appl. Mater. Today, 2017, 9, 259-265.

24. M. Ongaro, J. Roche, A. Kuhn and P. Ugo, ChemElectroChem, 2014, 1, 2048-2051.

25. A. Kirillova, C. Marschelke and A. Synytska, ACS Appl. Mater. Interfaces, 2019, 11, 9643-9671.

26. A. Chauhan, M. Rastogi, P. Scheier, C. Bowen, R. Kumar and R. Vaish, Appl. Phys. Rev., 2018, $5,041111$.

27. Z. W. Seh, S. Liu, M. Low, S.-Y. Zhang, Z. Liu, A. Mlayah and M.-Y. Han, Adv. Mater., 2012, 24, 2310-2314.

28. S. Tiewcharoen, C. Warakulwit, V. Lapeyre, P. Garrigue, L. Fourier, C. Elissalde, S. Buffière, P. Legros, M. Gayot, J. Limtrakul and A. Kuhn, Angew. Chem. Int. Ed., 2017, 56, 11431-11435.

29. B. Gupta, B. Goudeau and A. Kuhn, Angew. Chem. Int. Ed. 2017, 56, 14183-14186.

30. L. Bouffier, D. Zigah, N. Sojic, A. Kuhn, in Electroanalytical Chemistry: A Series of Advances, ed. A. J. Bard and C. G. Zoski, CRC Press 2017, vol. 27, ch. 2, pp. 27-118.

31. L. Li, P. A. Salvador and G. S. Rohrer, Nanoscale, 2014, 6, 24-42.

32. J. Zhao and F. E. Osterloh, J. Phys. Chem. Lett., 2014, 5, 782-786.

33. D. Adams, L. Brus, C. Chidsey, S. Creager, C. Creutz, C. Kagan, P. Kamat, M. Lieberman, S. Lindsay, R. Marcus, R. Metzger, M. Michel-Beyerle, J. Miller, M. Newton, D. Rolison, O. Sankey, K. Schanze, J. Yardley and X. Zhu, J. Phys. Chem. B, 2003, 34, 6668-6697..

34. V. Singh, S. Mohan, G. Singh, P. C. Pandey and R. Prakash, Sensor Actuat. B-Chem., 2008, 132, 99-106.

35. X. Yuan, Y. Xu, H. Meng, Y. Han, J. Wu, J. Xu and X. Zhang, Sep. Purif. Technol., 2018, 193, 358-367 




Scheme 1 a) Open bipolar electrochemistry set-up for the asymmetric electrodeposition of $\mathrm{Au}$ on a $\mathrm{TiO}_{2}$ pellet in the simutaneous presence of an electric field and UV light. Electrons are travelling from the $\delta^{-}$to the $\delta^{+}$side of the semiconductor b) Illustration of the buit-in band bending and charge separation in the caseof a semiconductor with a heterojunction c) Charge carrier flow in the case of materials without heterojunction. Red: positive feeder electrode; Blue: negative feeder electrode. $\delta^{+}$and $\delta^{-}$indicate the external polarization of $\mathrm{TiO}_{2}$.

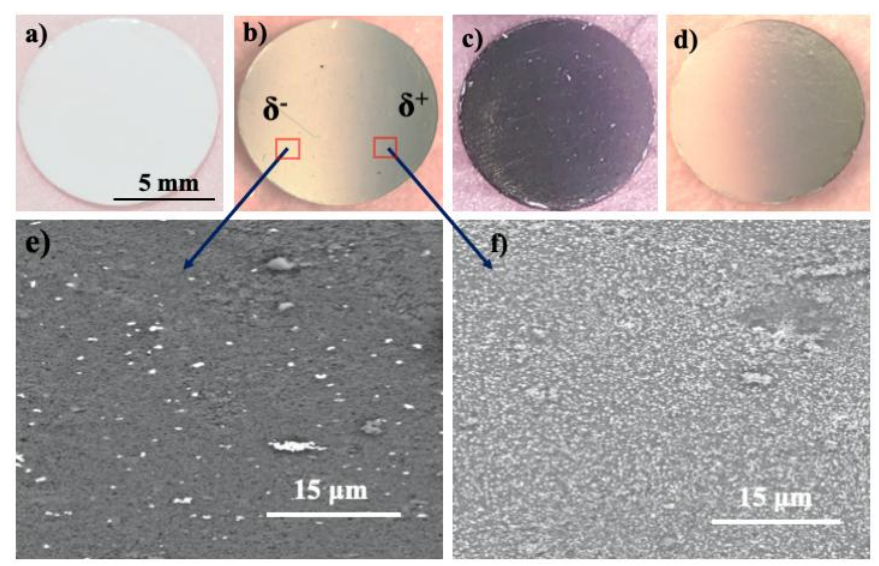

Figure 1 Bipolar photoelectrodeposition of Au particles on the surface of $\mathrm{TiO}_{2}$ pellets under different experimental conditionsa) $0 \%$ UV light, $90 \mathrm{~V} / \mathrm{cm}$ b) $1 \%$ UV light, $90 \mathrm{~V} / \mathrm{cm}$ and c) $1 \%$ UV light and $0 \mathrm{~V} / \mathrm{cm}$ d) $5 \%$ UV light, $120 \mathrm{~V} / \mathrm{cm}$. SEM images of selected areas at the surface of the pellet e) The $\mathrm{TiO}_{2}$ surface at the $\delta$ - side shows no significant metal depostion f) High density of Au nanoparticles deposited at the $\delta^{+}$extremity. The diameter of the $\mathrm{TiO}_{2}$ pellets is $1 \mathrm{~cm}$. $\left[\mathrm{AuCl}_{4}\right]=2 \mathrm{mM}$. Electrodeposition time of $30 \mathrm{sec}$.

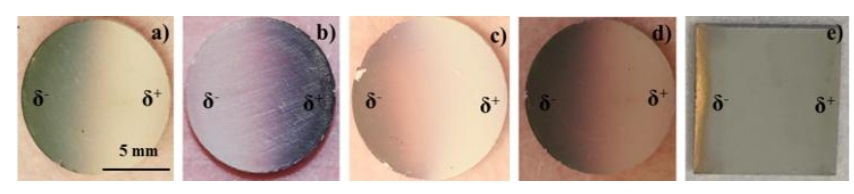

Figure 2 Bipolar photoelectrodepostion of Au on the surface of $\mathrm{TiO}_{2}$ pellets with different crystallographic composition and other semiconductors a) A-TiO $\mathrm{T}_{2}$ b) $\mathrm{AR}-\mathrm{TiO}_{2}$ (50:50\% ratio) c) $\mathrm{R}-\mathrm{TOO}_{2}$ d) $\mathrm{Ta}_{2} \mathrm{O}$, e) doped silicon. Relative light intensity $1 \%$; [AuCla] $=2 \mathrm{mM}, 30 \mathrm{sec}$ electrodeposition time. 


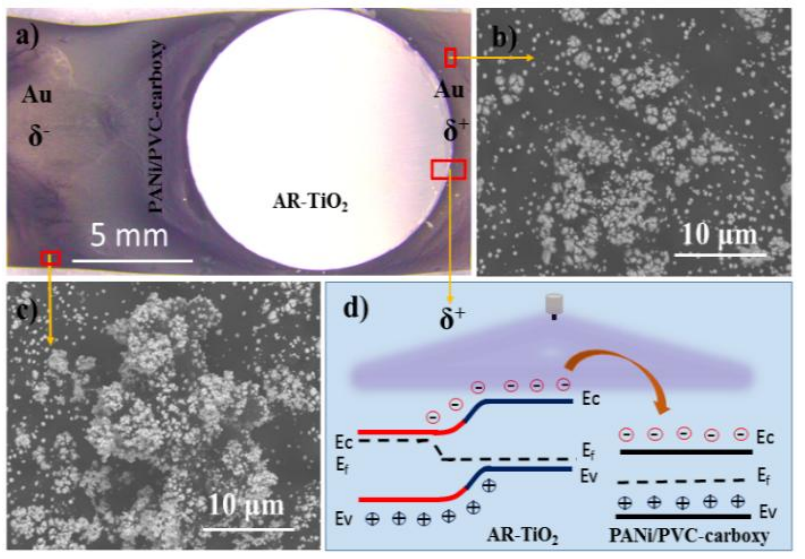

Figure 3 a) Optical image of the PANi/PVC-Carboxy/AR-TiO 2 hybrid after bipolar electro-depostion. b) and c) SEM images of selected spots on the hybrid object at the $\delta^{+}$and $\delta^{-}$extremitiesrespectively. d) Schematic illustration of energy levels of the components constituting the hybrid object at the $\delta^{+}$junction (red rectangle). $40 \mathrm{~V} / \mathrm{cm},[\mathrm{AuCl}]]=2 \mathrm{mM}, 30$ sec electrodeposition time. 